\title{
Prognostic impact of AJCC response criteria for neoadjuvant chemotherapy in stage II/III breast cancer patients: breast cancer subtype analyses
}

Yaewon Yang ${ }^{1,2}$, Seock-Ah Im $^{1,2^{*}}$, Bhumsuk Keam ${ }^{1,2}$, Kyung-Hun Lee ${ }^{1,2}$, Tae-Yong Kim ${ }^{1,2^{*}}$, Koung Jin Suh ${ }^{1,2}$, Han Suk Ryu ${ }^{2,4}$, Hyeong-Gon Moon ${ }^{2,3}$, Sae-Won Han ${ }^{1,2}$, Do-Youn Oh ${ }^{1,2}$, Wonshik Han ${ }^{2,3}$, Tae-You Kim ${ }^{1,2}$, In Ae Park ${ }^{2,4}$ and Dong-Young Noh ${ }^{2,3}$

\begin{abstract}
Background: Neoadjuvant chemotherapy (NAC) is a standard treatment for stage II/III breast cancer patients, and response to NAC is a useful prognostic marker. Since its introduction, 6-8 cycles of NAC has become the standard regimen to improve the outcome of these patients. The purpose of this study is to evaluate the prognostic impact of the American Joint Committee on Cancer (AJCC) response criteria and this tool's usefulness in four different breast cancer subtypes.

Methods: We conducted a retrospective cohort study of clinical stage II/III breast cancer patients who received NAC of more than 6 cycles. Response after NAC and the clinicopathological factors were reviewed. AJCC response criteria for NAC were adopted from the AJCC Manual, 7th edition: complete response (CR), partial response (PR), and no response (NR).

Results: A total of 183 patients were enrolled; 22 (12.0\%), 123 (67.2\%), and 38 (20.8\%) patients showed CR, PR, and NR, respectively. The AJCC response was significantly associated with relapse-free survival (RFS) $(P<0.001)$, whereas pathologic $C R(p C R)$, the current gold standard for response evaluation for NAC, was not $(P=0.140)$. AJCC response was a significant prognostic factor for RFS in all four breast cancer subtypes, namely luminal $A(P=0.006)$, luminal $\mathrm{B}(P=0.001)$, HER-2 enriched $(P=0.039)$, and triple-negative breast cancer $(P=0.035)$.

Conclusions: The AJCC response criteria represent a simple and easily reproducible tool for response evaluation of NAC patients and a useful clinical prognostic marker for RFS. These criteria also have a prognostic impact in all four breast cancer subtypes, including luminal A in which pCR has a limited role.
\end{abstract}

Keywords: Stage II or III, Breast neoplasm, Neoadjuvant chemotherapy, American Joint Committee on Cancer (AJCC) response, Relapse-free survival, Breast cancer subtype

\footnotetext{
* Correspondence: moisa@snu.ac.kr; ktyongmd@gmail.com

${ }^{1}$ Department of Internal Medicine, Seoul National University Hospital, Seoul

National University College of Medicine, 101 Daehak-ro, Jongno-gu, Seoul

110-744, Korea

Full list of author information is available at the end of the article
} 


\section{Background}

Breast cancer is the most common cancer in women worldwide, and 1.68 million cases are newly diagnosed annually [1]. It is the second most common cancer in females in South Korea, where 15,942 women were newly diagnosed in 2011 [2]. About 44\% of the newly diagnosed breast cancer patients are initially stage II or III, and neoadjuvant chemotherapy (NAC) or primary systemic therapy has become the standard treatment for this population $[3,4]$. Response to NAC is known to be useful in prognostic and predictive aspects. Pathologic complete response (pCR) is the most useful surrogate marker for overall survival in the NAC setting [5-7]. However, despite its clinical usefulness, pCR alone has limitations in evaluating residual disease after NAC. Recent pooled analysis of neoadjuvant clinical trials revealed that $\mathrm{pCR}$ is not a surrogate end-point marker for survival in the overall population because response to NAC is heterogeneous among breast cancer subgroups [8]. Several methods have been devised to evaluate the response to NAC, one of which is the American Joint Committee on Cancer (AJCC) response criteria for NAC [9]. Keam et al. [10] validated AJCC response criteria for NAC in 398 patients who received 3 cycles of doxorubicin plus docetaxel, and found these criteria to be useful in evaluating the response to NAC as well as predicting survival after short-course NAC.

Since the middle of the last decade the importance of pCR achievement has been emphasized, and to obtain higher rates of pCR extended cycles of neoadjuvant chemotherapy have been introduced [11, 12]. Six to eight cycles of NAC has recently become the standard treatment in clinical practice. In this study, we evaluated the clinical impact of AJCC response criteria in patients undergoing six or more cycles of NAC. In addition, we evaluated the clinical usefulness and prognostic value of the AJCC criteria in four different breast cancer subgroups [13].

\section{Methods}

\section{Study population and treatment}

We conducted a retrospective cohort study of the patients who received NAC in Seoul National University Hospital. Between January 2009 and December 2010, all stage II/III breast cancer patients receiving NAC were screened.

Detailed eligibility criteria were as follows: (1) pathologically confirmed breast cancer by core needle biopsy; (2) clinical stage II or III; (3) presence of objective measurable lesion by Response Evaluation Criteria In Solid Tumors (RECIST) version 1.1 [14]; (4) Eastern Cooperative Oncology Group performance status 0-2; (5) previously untreated; (6) cycles of neoadjuvant chemotherapy of 6 or more. Initial evaluation included physical examination, mammography, breast ultrasonography, chest computed tomography $(\mathrm{CT})$, bone scan, and breast magnetic resonance imaging (MRI). Initial tumor size was measured by MRI. Initial nodal staging was determined by physical examination and CT. After completing six or more cycles of neoadjuvant chemotherapy before definitive surgery, the patients were reexamined for response evaluation. Thereafter, the patients received curative surgery followed by adjuvant chemotherapy according to the physician's decision, considering response to NAC and final pathologic stage [15]. Patients received additional adjuvant radiation therapy [16], trastuzumab [17, 18], and hormonal therapy [19], if indicated.

The study protocol was reviewed and approved by the Institutional Review Board at the Seoul National University Hospital (H-0510-506-159). Recommendations of the Declaration of Helsinki for biomedical research involving human subjects were also followed.

\section{Response evaluation}

For evaluation of radiologic response, we obtained ultrasonography and MRI for primary breast cancer and chest CT for lymph node evaluation before and after NAC. The radiologic response was evaluated by RECIST criteria version 1.1 [14]. The initial clinical and postNAC pathologic staging was based on the AJCC Cancer Staging Manual, 7th edition. The details of AJCC response criteria for NAC were as follows [9].

(1)Complete response (CR) is defined as the absence of invasive carcinoma in the breast and lymph nodes. Residual in situ cancer, in the absence of invasive disease, constitutes a CR. Patients with isolated tumor foci in lymph nodes are not classified as having a $\mathrm{CR}$.

(2) Partial response (PR) is defined as a decrease in either or both $\mathrm{T}$ or $\mathrm{N}$ stage compared to the pretreatment $\mathrm{T}$ or $\mathrm{N}$, and no increase in either $\mathrm{T}$ or N. After chemotherapy, one should use the method that most clearly defined tumor dimensions at the baseline for this comparison, although prechemotherapy pT cannot be measured.

(3) No response (NR) is defined as no apparent change in either the $\mathrm{T}$ or $\mathrm{N}$ categories compared to the clinical pretreatment assignments, or increase in either the $\mathrm{T}$ or $\mathrm{N}$ categories at the time of pathologic evaluation.

Pathologic complete response (pCR) is defined as complete disappearance of invasive carcinoma, in both the breast and the axillary lymph nodes, after NAC. Residual ductal carcinoma in situ (DCIS) was included in the pCR category. 


\section{Clinicopathological examination}

The clinical characteristics (age at diagnosis, date of diagnosis, date, cycles and regimen of neoadjuvant chemotherapy, date of surgery, adjuvant therapy, date of last visit, date of relapse) and the laboratory test results (follicle-stimulating hormone, luteinizing hormone, and estradiol levels at diagnosis for determination of menopausal status [20]) were obtained by retrospective review of electronic medical records. We performed immunohistochemistry (IHC) using tissues obtained at diagnosis. Estrogen receptor (ER), progesterone receptor (PR), human epidermal growth factor receptor 2 (HER2), p53, $\mathrm{Bcl}-2$, epidermal growth factor receptor (EGFR), and Ki67 expression were evaluated. IHC was performed as previously described in our center's study series [21-24]. In the case of HER2 IHC 2+, fluorescence in situ hybridization (FISH) was performed to determine HER2 positivity. Positivity thresholds for classification were $\mathrm{ER} \geq 1 \%$; PR $\geq 1 \%$ [25]; HER2 = IHC 3+ (>10 \% invasive tumor cells with intense and circumferential membrane staining) and/or FISH positive (HER2/CEP17 ratio $\geq 2.2$ ) [26, 27]; and p53 $\geq 25 \%[22,28]$. The Ki-67 threshold of high $(\geq 14 \%)$ was based on work by Cheang et al., in which $14 \%$ best discriminated between luminal-A and luminal-B tumors [29].

\section{Breast cancer subtypes}

Breast cancer is further classified into several groups according to their molecular alteration, cellular composition, and clinical outcome. Tumor classification is useful in determining and predicting response to treatment as well as providing prognostic information. In this study, we classified breast cancer patients into four subgroups, namely luminal A (LA), luminal B (LB), HER2 enriched (HER2), and triple-negative breast cancer (TNBC), these definitions being mainly adopted from the 2011 St Gallen Consensus Panel [30]. Definitions of each subgroup are as follows.

(1)LA (highly endocrine responsive): ER positive, PR positive, HER2 negative, and Ki-67 low. The few ER-negative/PR-positive cases were considered ERpositive/PR-positive.

(2)LB (moderately endocrine responsive): ER positive and PR negative independent of other parameters, or ER positive, PR positive and at least one of grade 3, HER2 positive, and/or Ki-67 high.

(3) HER2: ER negative, PR negative, and HER2 positive.

(4)TNBC: ER negative, PR negative, and HER2 negative regardless of the expression of EGFR and basal cytokeratins.

Although there is some controversy surrounding endocrine therapy for patients with low ER-expressing tumors (1-10\%, weakly positive) regarding the benefit of tamoxifen and other endocrine therapies on survival and their relatively low toxicities, the American Society of Clinical Oncology (ASCO) and the College of American Pathologists (CAP) Panel recommended that ER and PR assays be considered positive if there are at least $1 \%$ positive tumor nuclei in the sample $[25,31,32]$. The recent National Comprehensive Cancer Network guideline for breast cancer also adopted the ASCO/CAP recommendation. We adopted the ASCO/ CAP guideline to determine the hormone receptorpositive tumors.

\section{Statistical analysis}

Relapse-free survival (RFS) was determined as the interval between the initiation of neoadjuvant chemotherapy and the date when disease relapse or progression was first documented, or the date of death from any cause. Local, regional, and distant relapse were all included in disease relapse, and contralateral breast cancer was not regarded as relapse. The Kaplan-Meier product limit method and the Cox proportional hazards regression model were used for survival analyses. Log-rank tests were used to compare RFS between different subgroups. Differences between breast cancer subtypes with regard to clinicopathologic characteristics were examined using 1-way analysis of variance (ANOVA) for the continuous variables (age, pre- and post-NAC tumor size), and $\chi^{2}$ tests for the remaining variables. All statistical tests were two-sided, with the level of significance established at $P<0.05$. All statistical analyses were carried out using SPSS version 21.0 (SPSS, Chicago, IL, USA).

\section{Results}

\section{Patients and treatment}

During the study period, 249 stage II/III breast cancer patients received NAC in Seoul National University Hospital. Sixty-six patients were excluded because they received less than six cycles of NAC, and finally 183 patients of median age 46 (range 25-71) years were enrolled and evaluated. The median follow-up duration was 38.0 (range 9-53) months. At the data cut-off point in June 2013, 41 patients (22.4 \%) had developed recurrent disease. The median RFS was not reached at the time. One hundred and fifty-three patients $(83.6 \%)$ were stage III. One hundred and nine (59.6\%) were premenopausal, and 106 (57.9\%) had hormone receptor-positive tumors. The majority of the patients received both anthracycline- and taxane-containing NAC. A total of 128 (69.9\%) received a concurrent anthracycline and taxane regimen, and $47(25.7 \%)$ received sequential anthracycline and taxane. Ten patients $(32.3 \%$ of HER2-positive patients) received a HER2-targeted agent (trastuzumab or trastuzumab emtansine)-containing NAC 
regimen. The baseline characteristics of the 183 patients are summarized in Table 1.

\section{Response to the neoadjuvant chemotherapy and relapse-free survival}

The results of the response evaluation after NAC according to the AJCC response criteria are shown in

Table 1 Baseline characteristics

\begin{tabular}{|c|c|c|}
\hline \multirow[t]{2}{*}{ Variables } & \multicolumn{2}{|c|}{ Number of patients $(N=183)$} \\
\hline & No & $\%$ \\
\hline Age, median (range) & $46(25-71)$ & \\
\hline \multicolumn{3}{|l|}{ Histology } \\
\hline Invasive ductal carcinoma & 167 & 91.3 \\
\hline Premenopausal/Postmenopausal & $109 / 74$ & $59.6 / 40.4$ \\
\hline \multicolumn{3}{|l|}{ Regimen of Neoadjuvant Chemotherapy } \\
\hline Concurrent anthracycline + taxane & 128 & 69.9 \\
\hline Sequential anthracycline + taxane & 47 & 25.7 \\
\hline $\begin{array}{l}\text { HER-2 directed agent containing } \\
\text { regimen }\end{array}$ & 10 & 5.5 \\
\hline Doxorubicin plus cyclophosphamide & 1 & 0.5 \\
\hline \multicolumn{3}{|l|}{ Type of Surgery } \\
\hline Breast conserving surgery & 104 & 56.8 \\
\hline Mastectomy & 79 & 43.2 \\
\hline \multicolumn{3}{|l|}{ Initial Clinical Stage } \\
\hline$\| \mathrm{A}$ & 3 & 1.6 \\
\hline$\| B$ & 27 & 14.8 \\
\hline$\| \mathrm{A}$ & 94 & 51.4 \\
\hline$\| I B$ & 26 & 14.2 \\
\hline$\| I I C$ & 33 & 18.0 \\
\hline \multicolumn{3}{|l|}{$\begin{array}{l}\text { Hormone receptor and HER2 expression } \\
\text { status }\end{array}$} \\
\hline Hormone receptor $(+)$ & 106 & 57.9 \\
\hline HER2 (+) & 58 & 31.7 \\
\hline \multicolumn{3}{|l|}{ Pathologic Stage } \\
\hline ур0 & 22 & 12.0 \\
\hline yplA & 41 & 22.4 \\
\hline ypllA & 42 & 23.0 \\
\hline ypllB & 22 & 12.0 \\
\hline ypllIA & 38 & 20.8 \\
\hline yplllB & 2 & 1.1 \\
\hline ypllIC & 16 & 8.7 \\
\hline \multicolumn{3}{|l|}{ Adjuvant therapy } \\
\hline Radiation therapy & 158 & 86.3 \\
\hline Chemotherapy & 62 & 33.9 \\
\hline Trastuzumab & 55 & $\begin{array}{l}94.8 \% \text { of patients with } \\
\text { HER2 positive tumor }\end{array}$ \\
\hline Hormonal therapy & 103 & $\begin{array}{l}97.1 \% \text { of patients with } \\
\text { HR positive tumor }\end{array}$ \\
\hline
\end{tabular}

HER2 human epidermal growth factor receptor 2, TNBC triple negative breast cancer
Table 2. Among 183 patients there were 22 with $\mathrm{CR}$ (12.0\%), 123 with PR (67.2\%), and 38 with NR (20.8\%). The 3-year RFS rates were $90.9 \%$ in CR, $79.9 \%$ in PR, and $48.5 \%$ in NR patients (Fig. 1, log-rank $P<0.001$ ). AJCC response was significantly associated with RFS (the hazard ratio (HR) for relapse of CR/PR group vs. NR group was 0.269 , with $95 \%$ confidence interval (CI) of $0.141-0.513, P<0.001)$. Figure 2 shows the hazard rate of relapse at the specific time from diagnosis (months) according to the three AJCC response groups. In the NR group, about $27 \%$ of patients relapsed within 1 year and $52 \%$ relapsed within the first 3 years from diagnosis, even during the adjuvant chemotherapy, radiotherapy, or hormone therapy. The AJCC response $\mathrm{CR}$ and PR groups showed similar prognosis and no statistical difference of RFS probability (HR of PR group 2.067, $95 \%$ CI 0.485-8.803, $P=0.326$ ), but the PR and NR groups showed a significant difference in RFS probability (HR of NR group 3.665, $95 \%$ CI 1.8797.146, $P<0.001)$. After adjusting for potential prognostic factors, the AJCC response was independently associated with RFS ( $P=0.004$, Table 3$)$, as well as the traditional prognostic factors such as pathologic stage and hormone receptor positivity. The $\mathrm{pCR}$ was not a significant predictor of RFS (Fig. 3, log-rank $P=0.110$ ), despite the Kaplan-Meier curve showing a tendency for survival difference between the two groups.

\section{Breast cancer subgroup analysis}

We divided the patients into four subgroups (LA, LB, HER2, and TNBC) as previously described, comprising 44 (24.1\%), 62 (33.9 \%), 31 (16.9\%), and 46 (25.1\%) patients, respectively. The rate of pCR was higher in TNBC (19.6 \%), HER2 (16.1\%), and LB (12.9\%) groups than in the LA (0 \%) group. The rate of AJCC response of CR plus PR was also higher in the TNBC (82.6 \%), HER2 (87.1\%), and LB $(79.0 \%)$ groups than in the LA $(70.5 \%)$ group. The response to the NAC according to the AJCC criteria was a significant prognostic factor for RFS in all four subgroups: LA $(P=0.006)$, LB $(P=0.001)$, HER2 $(P=0.039)$, and TNBC $(P=0.035)$ (Fig. 4 , log-rank test). In all subgroups, $C R$ and $P R$ patients showed a similar hazard rate of relapse: HR of PR patients was not assessable (no CR in LA group), $26.953(P=0.618)$, $2.690(P=0.345)$, and $1.613(P=0.663)$ in LA, LB, HER2,

Table 2 AJCC response after neoadjuvant chemotherapy

\begin{tabular}{llc}
\hline AJCC response & \multicolumn{2}{l}{ Number of patients $(N=183)$} \\
\cline { 2 - 3 } & Number & Percent $(\%)$ \\
\hline CR & 22 & 12.0 \\
PR & 123 & 67.2 \\
NR & 38 & 20.8 \\
\hline
\end{tabular}

$C R$ complete response, $P R$ partial response, $N R$ no response 


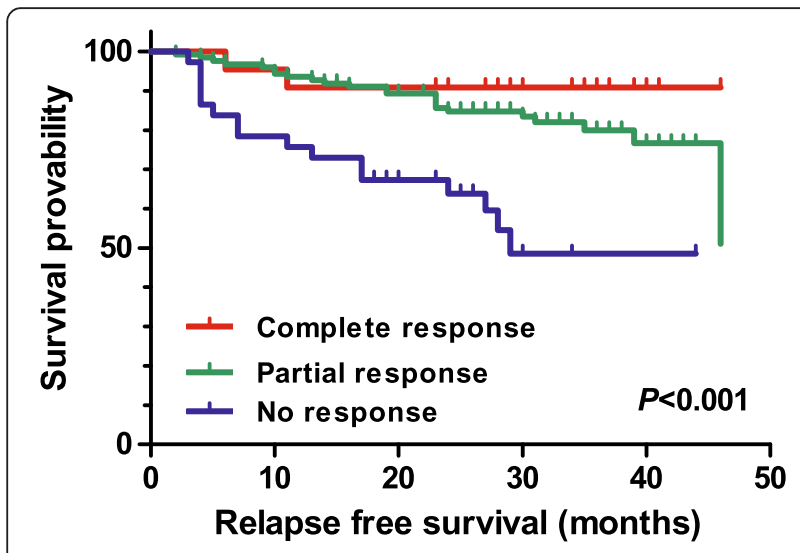

Fig. 1 Relapse-free survival probability according to the American Joint Committee on Cancer (AJCC) response, complete response, partial response, and no-response groups

and TNBC groups, respectively. Interestingly, NR patients showed a significantly higher risk of relapse than PR patients in all the four breast cancer subgroups: HR of NR patients was 12.898 (95\% CI 1.285-129.437, $P=0.030$ ), 4.224 (95 \% CI 1.088-16.394, $P=0.037$ ), 5.044 (95 \% CI $1.555-16.366, P=0.007$ ), and 4.206 (95 \% CI 1.12115.786, $P=0.033$ ) in the LA, LB, HER2, and TNBC groups, respectively. On the contrary, $\mathrm{pCR}$ was not significantly associated with RFS in any subgroups (Table 4). Pathologic stage was significantly associated with RFS in HER2 and TNBC patients, but not in the LA and LB groups. Table 4 shows the prognostic factors for RFS in each breast cancer subgroup by Cox regression.

\section{Discussion}

Response to the neoadjuvant chemotherapy in stage II or stage III breast cancer has a prognostic impact on RFS and overall survival [33-35]. pCR is the current standard criterion for evaluation of the response after

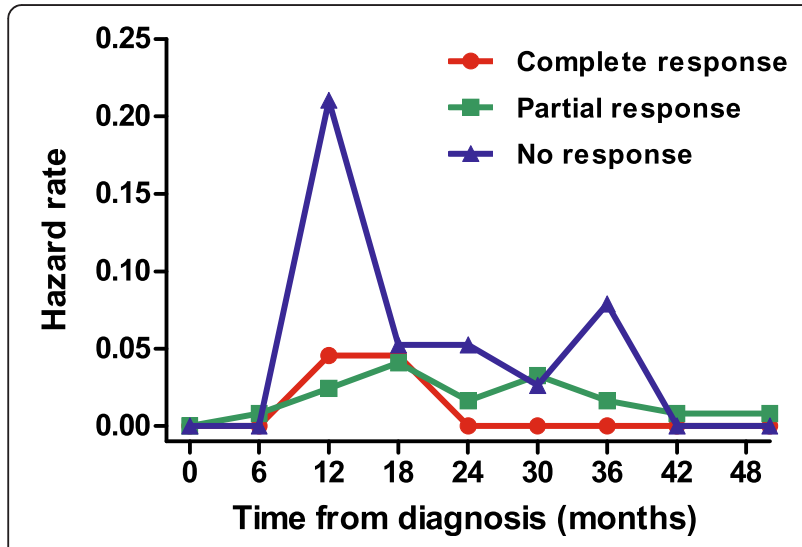

Fig. 2 Hazard rates of relapse of breast cancer patients after diagnosis according to the AJCC response criteria
NAC [5-7]. Addition of preoperative taxanes to doxorubicin and cyclophosphamide (AC) increased the proportion of patients with $\mathrm{pCR}$ compared with preoperative AC alone (26\% vs. $13 \%$, respectively; $P<0.001)$ [11, 36]. Following publication of the results of NSABP B-27 together with B-18 and several other studies, extended NAC to obtain a higher $\mathrm{pCR}$ rate became the standard treatment in clinical practice [11, 12, 36, 37]. Despite its clinical usefulness, pCR has some limitations as a prognostic and predictive marker, and several groups proposed new methods for grouping post-NAC patients to evaluate the response to NAC $[35,38,39]$, one of which is the AJCC response criteria for NAC. A previous study by Keam et al. [10] showed that the AJCC response criteria for NAC correlate well with radiologic response criteria and have a prognostic value for both RFS and overall survival in patients with three cycles of concurrent doxorubicin plus docetaxel neoadjuvant chemotherapy. CR, $\mathrm{PR}$, and NR rates were 9.8, 59.3, and $30.7 \%$, 5-year RFS 89.6, 74.1, and $62.6 \%(P=0.002)$, and 5-year overall survival 97.4, 88.6, and $78.3 \%(P=0.012)$, respectively [10]. In the current study, we demonstrated that the AJCC response criteria represent a useful prognostic factor for RFS in patients undergoing 6 or more cycles of NAC. The rates of $C R$ and $P R$ are significantly higher (12 and $67.2 \%$, respectively) than those from 3 cycles of NAC in the previous study [10]. Because the follow-up duration is short, the prognostic impact of AJCC response with extended NAC on the overall survival has not yet been obtained.

Previous studies reported different rates of $\mathrm{pCR}$ after NAC between breast cancer subgroups, and suggested the clinical usefulness of $\mathrm{pCR}$ as a surrogate marker of survival is different in each breast cancer subtype [13]. According to von Minckwitz et al., pCR is a suitable surrogate end point for patients with LB/HER2-negative, HER2-positive (non-luminal), and triple-negative disease but not for those with LB/HER2-positive or LA tumors [13]. LA is a slowly proliferating tumor type, whose response to NAC is not as good as that in highly proliferating tumor types. Given these heterogeneous responses to NAC, a recent meta-analysis revealed that $\mathrm{pCR}$ alone is not sufficient as a surrogate end point for event-free survival and overall survival in the general breast cancer population [8]. In fact, no patients achieved pCR in the LA group in our current study. Furthermore, in contrast to previous studies, pCR was not associated with RFS in any of our subgroups. This might result from a short follow-up period and a lack of sufficient events (relapse) for obtaining statistical power. By contrast, our analyses of the four breast cancer subtypes demonstrated that AJCC response after NAC was a significant prognostic marker for RFS in all four breast cancer subgroups, even in LA patients. 
Table 3 Univariate and multivariate analyses for relapse free survival

\begin{tabular}{|c|c|c|c|c|c|c|}
\hline \multirow[t]{2}{*}{ Variables } & \multicolumn{3}{|c|}{ Univariate analysis } & \multicolumn{3}{|c|}{ Multivariate analysis } \\
\hline & $\mathrm{HR}$ & $95 \% \mathrm{Cl}$ & $P$ & $\mathrm{HR}$ & $95 \% \mathrm{Cl}$ & $P$ \\
\hline Age ( $\leq$ median) & 0.685 & $0.364-1.287$ & 0.240 & & - & \\
\hline Menopausal status (Premenopausal) & 0.846 & $0.449-1.594$ & 0.605 & & - & \\
\hline $\mathrm{pCR}$ & 0.342 & $0.082-1.420$ & 0.140 & & - & \\
\hline AJCC response (CR plus PR) & 0.309 & $0.172-0.556$ & 0.000 & 0.374 & $0.192-0.728$ & 0.004 \\
\hline Surgery (BCS) & 0.428 & $0.228-0.803$ & 0.008 & 0.475 & $0.252-0.894$ & 0.021 \\
\hline Pathologic Stage (yp0,l) & 0.235 & $0.092-0.600$ & 0.002 & 0.267 & $0.094-0.756$ & 0.013 \\
\hline Hormone receptor positive & 0.447 & $0.236-0.846$ & 0.013 & 0.402 & $0.198-0.818$ & 0.012 \\
\hline HER2 positive & 1.059 & $0.761-1.474$ & 0.734 & & - & \\
\hline TNBC & 1.038 & $0.056-2.131$ & 0.919 & & - & \\
\hline Subtype (Luminal A) & 0.379 & $0.145-0.985$ & 0.047 & 0.314 & $0.103-0.962$ & 0.043 \\
\hline
\end{tabular}

There was no significant difference in RFS between pCR and non-pCR groups $(P=0.110$, log-rank test). The AJCC response in CR and PR patients showed a similar prognosis, but PR and NR groups showed a significant difference in RFS probability. We also compared the RFS of $\mathrm{CR}+\mathrm{PR}$ groups with that of the NR group using AJCC response criteria. The RFS of $C R+P R$ patients was significantly longer than that in the NR group (median RFS not reached vs. 29 months, $P<0.001$, logrank test). The RFS probability difference between the $\mathrm{CR}$ and PR groups was not significantly different, although the HR of PR patients was higher, at 2.067 (95\% CI $0.485-8.803, P=0.326)$. This might result from the small sample size and, thus, insufficient events (sample size 145 and 24 events at the data cut-off). The AJCC response criteria may thus represent a simple and easily applicable tool to evaluate residual disease and a new surrogate end point in neoadjuvant trials. Further follow-up is needed to confirm the prognostic impact of

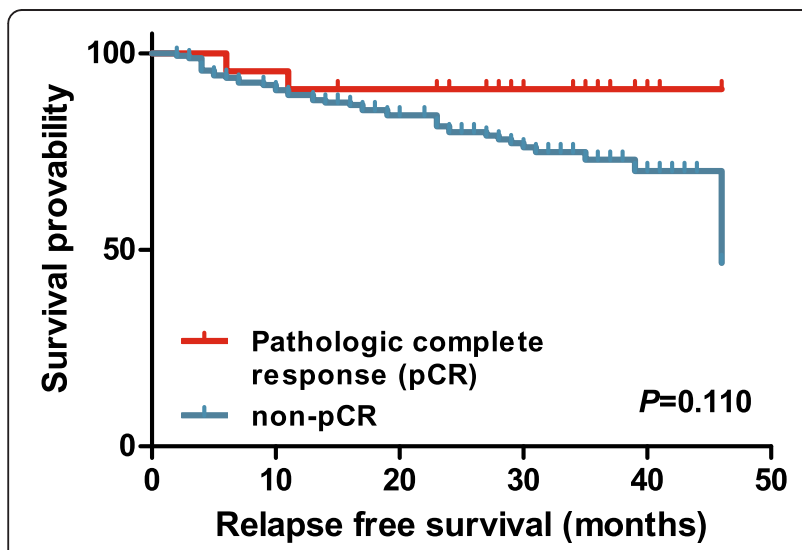

Fig. 3 Relapse-free survival probability according to the pathologic complete response the AJCC response criteria and $\mathrm{pCR}$ in each breast cancer subgroup.

We further analyzed the LA patients. In our hospital's NAC cohort, we were able to obtain the clinicopathologic and survival data of all the LA patients, regardless of the number of NAC cycles ( $<6$ or $\geq 6$ cycles). For the LA patients, pCR was achieved in neither group. The AJCC response rate ( $C R+P R$ portion) was higher in the extended-NAC group than in the short-course NAC group (70.5 \% vs. $56.3 \%)$, although statistically not significant $(P=0.302)$. This trend is consistent with the previous report by Moon et al. [40], which showed that continuous tumor shrinkage occurred in their ERpositive tumor group during extended NAC, while tumor shrinkage mainly occurred in the early period of NAC in the ER-negative group. By Cox regression of prognostic factors on RFS in the LA subgroup, premenopausal patients showed a significantly lower risk of relapse than the postmenopausal patients (HR 0.092, $95 \%$ CI 0.009-0.906, $P=0.041$; Table 4). This benefit might result from the additional secondary ovarian function suppression effect of chemotherapeutic agents in premenopausal patients. All things considered, in younger LA patients in premenopausal status an extended-NAC strategy might be more beneficial.

From the hazard rate of RFS of the patients according to AJCC responses (Fig. 2), we discerned several clinical implications. In the NR group 27, 41, and $52 \%$ of the patients relapsed during first 1, 2, and 3 years after diagnosis, respectively. For these patients, thorough physical examination and work-up for locoregional and/or distant metastases should be performed even during adjuvant therapies. From these data, we may suggest the necessity of further adjuvant chemotherapy even after the use of both an anthracyclineand taxane-containing NAC regimen and the selection 

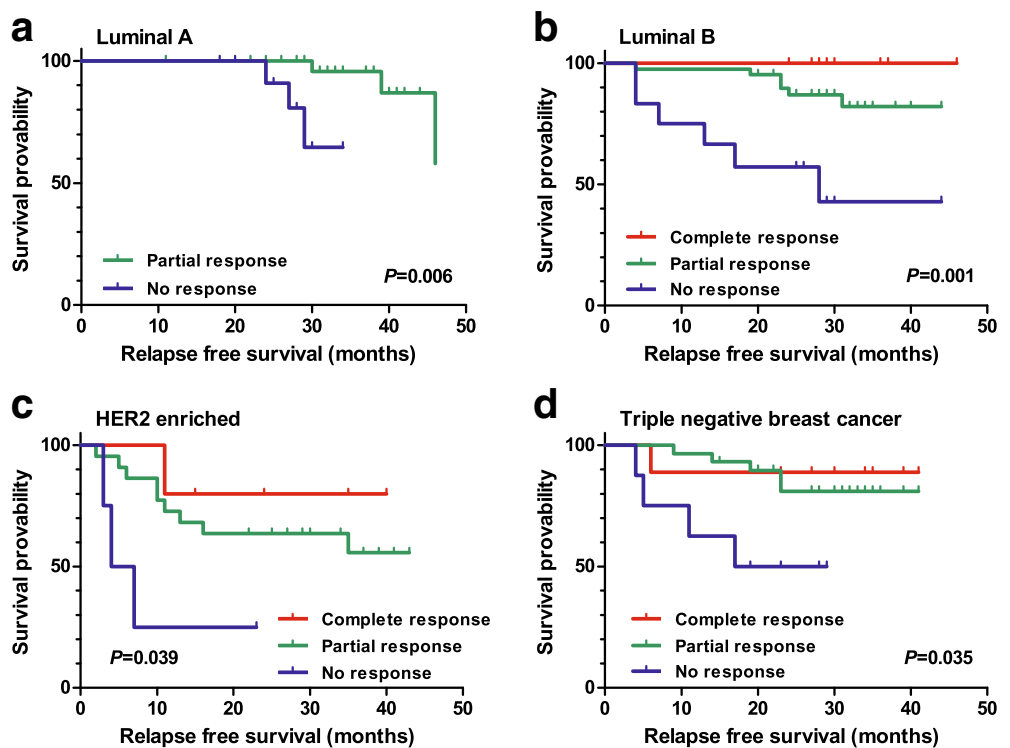

Fig. 4 Relapse-free survival probability according to the AJCC response in each breast cancer subgroup: a luminal A type, b luminal B type, c HER2-enriched type, and $\mathbf{d}$ triple-negative breast cancer type

of high-risk patients who require adjuvant chemotherapy. Ongoing adjuvant clinical trials (JBCRG04 (CREATE-X), NCT01864746 (PENELOPE-B)) are targeting high-risk patients with residual diseases after neoadjuvant chemotherapy. The recent results of the CREATE-X trial revealed that treatment with adjuvant capecitabine increased disease-free survival for patients with HER2negative breast cancer who had residual disease after neoadjuvant chemotherapy [41]. The data also showed a tendency toward improving overall survival, albeit

Table 4 Prognostic factors according to subgroup analysis

\begin{tabular}{|c|c|c|c|c|c|c|c|c|c|c|c|c|}
\hline \multirow[t]{2}{*}{ Variables } & \multicolumn{3}{|c|}{ Luminal A } & \multicolumn{3}{|c|}{ Luminal B } & \multicolumn{3}{|l|}{ HER2 } & \multicolumn{3}{|l|}{ TNBC } \\
\hline & $\overline{H R}$ & $95 \% \mathrm{Cl}$ & $P$ & $\overline{H R}$ & $95 \% \mathrm{Cl}$ & $P$ & $\overline{H R}$ & $95 \% \mathrm{Cl}$ & $P$ & $\overline{H R}$ & $95 \% \mathrm{Cl}$ & $P$ \\
\hline \multicolumn{13}{|l|}{$\overline{\text { Age }}$} \\
\hline$\leq$ median & 0.477 & $0.078-2.907$ & 0.422 & 0.646 & $0.207-2.019$ & 0.453 & 0.616 & $0.188-2.016$ & 0.423 & 0.597 & $0.168-2.115$ & 0.424 \\
\hline$>$ median & 1 & & & 1 & & & 1 & & & 1 & & \\
\hline \multicolumn{13}{|l|}{ Menopausal status } \\
\hline Premenopausal & 0.092 & $0.009-0.906$ & 0.041 & 0.875 & $0.282-2.727$ & 0.818 & 0.482 & $0.132-1.754$ & 0.268 & 0.741 & $0.214-2.558$ & 0.635 \\
\hline Postmenopausal & 1 & & & 1 & & & 1 & & & 1 & & \\
\hline $\mathrm{pCR}$ & & - & & 0.039 & $0.000-46.767$ & 0.370 & 0.360 & $0.047-2.773$ & 0.327 & 0.420 & $0.053-3.323$ & 0.411 \\
\hline vs non-pCR & & & & 1 & & & 1 & & & 1 & & \\
\hline \multicolumn{13}{|l|}{ AJCC response } \\
\hline CR plus PR & 0.078 & $0.008-0.778$ & 0.030 & 0.157 & $0.050-0.499$ & 0.002 & 0.221 & $0.058-0.838$ & 0.026 & 0.219 & $0.061-0.783$ & 0.020 \\
\hline NR & 1 & & & 1 & & & 1 & & & 1 & & \\
\hline \multicolumn{13}{|l|}{ Pathologic Stage } \\
\hline yp0,l & 0.517 & $0.247-1.081$ & 0.080 & 0.160 & $0.021-1.238$ & 0.079 & 0.162 & $0.036-0.732$ & 0.018 & 0.182 & $0.038-0.861$ & 0.032 \\
\hline ypll, III, IV & 1 & & & 1 & & & 1 & & & 1 & & \\
\hline \multicolumn{13}{|l|}{ Surgery } \\
\hline $\mathrm{BCS}$ & 0.414 & $0.069-2.493$ & 0.336 & 0.329 & $0.101-1.071$ & 0.065 & 0.296 & $0.081-1.081$ & 0.065 & 0.930 & $0.240-3.660$ & 0.917 \\
\hline Mastectomy & 1 & & & 1 & & & 1 & & & 1 & & \\
\hline
\end{tabular}

$p C R$ pathologic complete response, $C R$ complete response, $P R$ partial response, $N R$ no response, $B C S$ breast conserving surgery, $H E R 2$ human epidermal growth factor receptor 2, TNBC triple negative breast cancer, $H R$ hazard ratio, $95 \% C l 95 \%$ confidence interval, $P$ p-value 
statistically insignificant, which might derive from the short follow-up period.

Our study has some limitations. First, because of the retrospective design of the study, the probability existed of selecting patients with a good response to NAC (whose cancer did not progress during NAC) while excluding patients with early progression or non-response. The investigation of those who received a short course of NAC ( $<6$ cycles, total $n=66)$ provided some clues. They encompassed both groups who were initially scheduled to receive a short course of chemotherapy and who progressed during NAC. Although it was difficult to distinguish the two groups by retrospective medical record review, there was only one patient with definite clinical disease progression during NAC who received mastectomy after three cycles of NAC. According to the radiologic response criteria, there was no difference in the portion of patients with progressive disease ( $4.5 \%$ vs. $4.4 \%,<6$ cycles vs. $\geq 6$ cycles of NAC). Therefore, the selection bias is expected to be minimal in this study. Second, the follow-up duration in this study is short, so the prognostic impact of the AJCC response criteria on overall survival for a long course of NAC could not be demonstrated. Third, the sample sizes of the each breast cancer subgroup are rather small, so the prognostic impact of the AJCC response criteria in each breast cancer subtype should be investigated in a larger population in subsequent studies.

Despite these limitations, this is the first report to demonstrate the clinical usefulness of AJCC response criteria in patients undergoing six or more cycles of NAC with neoadjuvant chemotherapy regimens used in clinical practice. Furthermore, our analyses demonstrated that the AJCC response criteria represented a significant prognostic marker for RFS in all four breast cancer subgroups, including the LA subgroup in which pCR has a limited role. The AJCC response criteria serve as a simple and easily reproducible tool for response evaluation in breast cancer patients in the NAC setting in comparison with the classically used Residual Cancer Burden measurement method or Miller-Payne grading system [35, 38]. The AJCC response criteria could help overcome the limitations of $\mathrm{pCR}$, as they may be valid in all breast cancer subgroups and be helpful in selecting those high-risk patients who need further adjuvant treatment. In addition, we performed a preand post-NAC paired imaging study (breast MRI or chest $\mathrm{CT}$ with breast ultrasonography) for accurate clinical staging, examination of radiologic response, and evaluation of AJCC response. Further follow-up is needed to establish the potential prognostic role of the AJCC response criteria and other clinicopathologic markers of overall survival in the NAC setting of six to eight cycles.

\section{Conclusions}

The AJCC response criteria represent a simple and easily reproducible clinical tool for predicting RFS in patients with stage II/III breast cancer undergoing six or more cycles of neoadjuvant chemotherapy. It has a prognostic impact in all four breast cancer subtypes, including the LA group in which pCR has a limited role. The AJCC response to NAC could also be a useful tool for selecting high-risk patients who need further adjuvant chemotherapy and more thorough examination for relapse, together with classical prognostic markers such as pathologic stage and breast cancer subtypes.

\section{Abbreviations \\ AJCC, American Joint Committee on Cancer; Cl, confidence interval; CR, complete response; ER, estrogen receptor; HER2, human epidermal growth factor receptor $2 ; H R$, hazard ratio; $I H C$, immunohistochemistry; $L A$, luminal $A$; $L B$, luminal $B$; NAC, neoadjuvant chemotherapy; NR, no response; $P C R$, pathologic $C R$; PR, partial response; PR, progesterone receptor; RFS, relapse- free survival; TNBC, triple-negative breast cancer}

\section{Acknowledgements}

None.

\section{Funding}

This research was partly supported by a grant of the Korea Health Technology R\&D Project through the Korea Health Industry Development Institute (KHIDI), funded by the Ministry of Health \& Welfare, Republic of Korea (HI14C1277) and partly supported by Priority Research Centers Program through the National Research Foundation of Korea(NRF) funded by the Ministry of Education, Science and Technology(2009-0093820).

\section{Availability of data and materials}

Detailed data of the patients will not be shared at the moment since these data are used for further publications.

\section{Authors' contributions}

YY, BK, and SAI were involved in the study concept and design. Patient management and surgery were carried out by SAI, KHL, TYK1 ${ }^{*}, \mathrm{HGM}, \mathrm{SWH}$, DYO, WH, TYK2 ${ }^{*}$, and DYN. HSR and IAP were responsible for all matters regarding pathology. YY collected and evaluated clinical data. YY, SAI, TYK1, KJS, and KHL performed data interpretation and statistical analyses. YY drafted the manuscript. All authors contributed to data interpretation, review of the manuscript, and approval of the final version. (* The initial TYK1 stands for the author Tae-Yong Kim and the initial TYK2 stands for Tae-You Kim).

\section{Competing interests}

The authors declare that they have no competing interests.

\section{Consent for publication}

Not applicable.

\section{Ethics approval and consent to participate}

The study protocol was reviewed and approved by the Institutional Review Board at the Seoul National University Hospital (H-0510-506-159). Recommendations of the Declaration of Helsinki for biomedical research involving human subjects were also followed.

\section{Author details}

${ }^{1}$ Department of Internal Medicine, Seoul National University Hospital, Seoul National University College of Medicine, 101 Daehak-ro, Jongno-gu, Seoul 110-744, Korea. ${ }^{2}$ Cancer Research Institute, Seoul National University, Seoul, Korea. ${ }^{3}$ Department of Surgery, Seoul National University Hospital, Seoul National University College of Medicine, Seoul, Korea. ${ }^{4}$ Department of Pathology, Seoul National University Hospital, Seoul National University College of Medicine, Seoul, Korea. 
Received: 15 April 2015 Accepted: 4 July 2016 Published online: 21 July 2016

\section{References}

1. Ferlay J, Soerjomataram I, Dikshit R, Eser S, Mathers C, Rebelo M, et al. Cancer incidence and mortality worldwide: sources, methods and major patterns in GLOBOCAN 2012. Int J Cancer. 2015;136(5):E359-86. doi:10.1002/ ijc.29210.

2. Jung KW, Won YJ, Kong HJ, Oh CM, Lee DH, Lee JS. Cancer statistics in Korea: incidence, mortality, survival, and prevalence in 2011. Cancer Res Treat. 2014;46(2):109-23. doi:10.4143/crt.2014.46.2.109.

3. Korean Breast Cancer Society. Breast cancer facts and figures. 2013. http:// www.kbcs.or.kr/journal/file/2013_Breast_Cancer_Facts_and_Figures updated.pdf. Accessed 16 Oct 2013.

4. Kaufmann M. Recommendations from an International Expert Panel on the use of neoadjuvant (Primary) systemic treatment of operable breast cancer: an update. J Clin Oncol. 2006;24(12):1940-9. doi:10.1200/jco.2005.02.6187.

5. Bear HD, Anderson S, Smith RE, Geyer Jr CE, Mamounas EP, Fisher B, et al. Sequential preoperative or postoperative docetaxel added to preoperative doxorubicin plus cyclophosphamide for operable breast cancer: National Surgical Adjuvant Breast and Bowel Project Protocol B-27. J Clin Oncol. 2006;24(13):2019-27. doi:10.1200/jco.2005.04.1665.

6. Bonadonna G, Valagussa P, Brambilla C, Ferrari L, Moliterni A, Terenziani M, et al. Primary chemotherapy in operable breast cancer: eight-year experience at the Milan Cancer Institute. J Clin Oncol. 1998;16(1):93-100.

7. Fisher B, Brown A, Mamounas E, Wieand S, Robidoux A, Margolese RG, et al. Effect of preoperative chemotherapy on local-regional disease in women with operable breast cancer: findings from National Surgical Adjuvant Breast and Bowel Project B-18. J Clin Oncol. 1997;15(7):2483-93.

8. Cortazar P, Zhang L, Untch M, Mehta K, Costantino JP, Wolmark N, et al. Pathological complete response and long-term clinical benefit in breast cancer: the CTNeoBC pooled analysis. Lancet. 2014;384(9938):164-72. doi:10.1016/s0140-6736(13)62422-8.

9. Edge SB, Compton CC. The American Joint Committee on Cancer: the 7th edition of the AJCC cancer staging manual and the future of TNM. Ann Surg Oncol. 2010;17(6):1471-4. doi:10.1245/s10434-010-0985-4.

10. Keam B, Im S-A, Lim Y, Han S-W, Moon H-G, Oh D-Y, et al. Clinical usefulness of AJCC response criteria for neoadjuvant chemotherapy in breast cancer. Ann Surg Oncol. 2013;20(7):2242-9. doi:10.1245/s10434012-2756-x.

11. Smith IC, Heys SD, Hutcheon AW, Miller ID, Payne S, Gilbert FJ, et al. Neoadjuvant chemotherapy in breast cancer: significantly enhanced response with docetaxel. J Clin Oncol. 2002;20(6):1456-66.

12. von Minckwitz G, Kummel S, Vogel P, Hanusch C, Eidtmann H, Hilfrich J, et al. Intensified neoadjuvant chemotherapy in early-responding breast cancer: phase III randomized GeparTrio study. J Natl Cancer Inst. 2008;100(8):552-62. doi:10.1093/jnci/djn089.

13. von Minckwitz G, Untch M, Blohmer JU, Costa SD, Eidtmann H, Fasching PA, et al. Definition and impact of pathologic complete response on prognosis after neoadjuvant chemotherapy in various intrinsic breast cancer subtypes. J Clin Oncol. 2012;30(15):1796-804. doi:10.1200/jco.2011.38.8595.

14. Eisenhauer EA, Therasse P, Bogaerts J, Schwartz LH, Sargent D, Ford R, et al. New response evaluation criteria in solid tumours: revised RECIST guideline (version 1.1). Eur J Cancer. 2009;45(2):228-47. doi:10.1016/j.ejca.2008.10.026.

15. Loprinzi CL, Thome SD. Understanding the utility of adjuvant systemic therapy for primary breast cancer. J Clin Oncol. 2001;19(4):972-9.

16. Overgaard M, Hansen PS, Overgaard J, Rose C, Andersson M, Bach F, et al. Postoperative radiotherapy in high-risk premenopausal women with breast cancer who receive adjuvant chemotherapy. Danish Breast Cancer Cooperative Group 82b Trial. N Engl J Med. 1997;337(14):949-55. doi:10.1056/nejm199710023371401.

17. Moja L, Tagliabue L, Balduzzi S, Parmelli E, Pistotti V, Guarneri V, et al. Trastuzumab containing regimens for early breast cancer. Cochrane Database Syst Rev. 2012;4:Cd006243. doi:10.1002/14651858.CD006243.pub2.

18. Romond EH, Perez EA, Bryant J, Suman VJ, Geyer Jr CE, Davidson NE, et al. Trastuzumab plus adjuvant chemotherapy for operable HER2-positive breast cancer. N Engl J Med. 2005;353(16):1673-84. doi:10.1056/NEJMoa052122.

19. EBCTCG. Effects of chemotherapy and hormonal therapy for early breast cancer on recurrence and 15-year survival: an overview of the randomised trials. Lancet. 2005;365(9472):1687-717. doi:10.1016/s0140-6736(05)66544-0.
20. Burger HG, Dudley EC, Robertson DM, Dennerstein L. Hormonal changes in the menopause transition. Recent Prog Horm Res. 2002;57: 257-75.

21. Jung SY, Han W, Lee JW, Ko E, Kim E, Yu JH, et al. Ki-67 expression gives additional prognostic information on St. Gallen 2007 and Adjuvant! Online risk categories in early breast cancer. Ann Surg Oncol. 2009;16(5):1112-21. doi:10.1245/s10434-009-0334-7.

22. Keam B, Im SA, Kim HJ, Oh DY, Kim JH, Lee SH, et al. Prognostic impact of clinicopathologic parameters in stage II/III breast cancer treated with neoadjuvant docetaxel and doxorubicin chemotherapy: paradoxical features of the triple negative breast cancer. BMC Cancer. 2007;7:203. doi:10.1186/ 1471-2407-7-203.

23. Keam B, Im S-A, Lee K-H, Han S-W, Oh D-Y, Kim J, et al. Ki-67 can be used for further classification of triple negative breast cancer into two subtypes with different response and prognosis. Breast Cancer Res. 2011;13(2):R22. doi:10.1186/bcr2834

24. Lee KH, Im SA, Oh DY, Lee SH, Chie EK, Han W, et al. Prognostic significance of bcl-2 expression in stage III breast cancer patients who had received doxorubicin and cyclophosphamide followed by paclitaxel as adjuvant chemotherapy. BMC Cancer. 2007;7:63. doi:10.1186/1471-2407-7-63.

25. Hammond ME, Hayes DF, Dowsett M, Allred DC, Hagerty KL, Badve S, et al. American Society of Clinical Oncology/College of American Pathologists guideline recommendations for immunohistochemical testing of estrogen and progesterone receptors in breast cancer. J Clin Oncol. 2010;28(16):2784-95. doi:10.1200/JCO.2009.25.6529.

26. Wolff AC, Hammond ME, Schwartz JN, Hagerty KL, Allred DC, Cote RJ, et al. American Society of Clinical Oncology/College of American Pathologists guideline recommendations for human epidermal growth factor receptor 2 testing in breast cancer. J Clin Oncol. 2007;25(1):118-45. doi:10.1200/jco. 2006.09.2775.

27. Yaziji H, Goldstein LC, Barry TS, Werling R, Hwang H, Ellis GK, et al. HER-2 testing in breast cancer using parallel tissue-based methods. JAMA. 2004;291(16):1972-7. doi:10.1001/jama.291.16.1972.

28. Kim T, Han W, Kim MK, Lee JW, Kim J, Ahn SK, et al. Predictive significance of p53, Ki-67, and $\mathrm{BCl}-2$ expression for pathologic complete response after neoadjuvant chemotherapy for triple-negative breast cancer. J Breast Cancer. 2015;18(1):16-21. doi:10.4048/jbc.2015.18.1.16.

29. Cheang MC, Chia SK, Voduc D, Gao D, Leung S, Snider J, et al. Ki67 index, HER2 status, and prognosis of patients with luminal B breast cancer. J Nat Cancer Inst. 2009;101(10):736-50. doi:10.1093/jnci/djp082.

30. Goldhirsch A, Wood WC, Coates AS, Gelber RD, Thurlimann B, Senn HJ. Strategies for subtypes-dealing with the diversity of breast cancer: highlights of the St. Gallen International Expert Consensus on the Primary Therapy of Early Breast Cancer 2011. Ann Oncol. 2011;22(8):1736-47. doi:10.1093/annonc/mdr304.

31. Hammond ME. ASCO-CAP guidelines for breast predictive factor testing: an update. Appl Immunohistochem Mol Morphol. 2011;19(6):499-500. doi:10.1097/PAl.0b013e31822a8eac.

32. Allred DC, Carlson RW, Berry DA, Burstein HJ, Edge SB, Goldstein LJ, et al. NCCN task force report: estrogen receptor and progesterone receptor testing in breast cancer by immunohistochemistry. J Natl Compr Canc Netw. 2009;7 Suppl 6:S1-21. quiz S2-3.

33. Liedtke C, Mazouni C, Hess KR, Andre F, Tordai A, Mejia JA, et al. Response to neoadjuvant therapy and long-term survival in patients with triplenegative breast cancer. J Clin Oncol. 2008;26(8):1275-81. doi:10.1200/JCO. 2007.14.4147

34. Schott AF, Hayes DF. Defining the benefits of neoadjuvant chemotherapy for breast cancer. J Clin Oncol. 2012;30(15):1747-9. doi:10.1200/JCO.2011.41.3161.

35. Symmans WF, Peintinger F, Hatzis C, Rajan R, Kuerer $H$, Valero V, et al. Measurement of residual breast cancer burden to predict survival after neoadjuvant chemotherapy. J Clin Oncol. 2007;25(28):4414-22. doi:10.1200/ jco.2007.10.6823.

36. Vriens BEPJ, Aarts MJB, de Vries B, van Gastel SM, Wals J, Smilde TJ, et al. Doxorubicin/cyclophosphamide with concurrent versus sequential docetaxel as neoadjuvant treatment in patients with breast cancer. Eur J Cancer. 2013. doi:10.1016/j.ejca.2013.06.012.

37. Oakman C, Francis PA, Crown J, Quinaux E, Buyse M, De Azambuja E, et al. Overall survival benefit for sequential doxorubicin-docetaxel compared with concurrent doxorubicin and docetaxel in node-positive breast cancer-8year results of the Breast International Group 02-98 phase III trial. Ann Oncol. 2013;24(5):1203-11. doi:10.1093/annonc/mds627. 
38. Ogston KN, Miller ID, Payne S, Hutcheon AW, Sarkar TK, Smith I, et al. A new histological grading system to assess response of breast cancers to primary chemotherapy: prognostic significance and survival. Breast. 2003;12(5):320-7.

39. Rodenhuis S, Mandjes IA, Wesseling J, van de Vijver MJ, Peeters MJ, Sonke GS, et al. A simple system for grading the response of breast cancer to neoadjuvant chemotherapy. Ann Oncol. 2010;21(3):481-7. doi:10.1093/annonc/mdp348.

40. Moon HG, Im SA, Han W, Oh DY, Han SW, Keam B, et al. Estrogen receptor status confers a distinct pattern of response to neoadjuvant chemotherapy: implications for optimal durations of therapy: distinct patterns of response according to ER expression. Breast Cancer Res Treat. 2012;134(3):1133-40. doi:10.1007/s10549-012-2145-y.

41. Lee S, Toi M, Lee E, Ohtani S, Im Y, Im S, et al. A phase III trial of adjuvant capecitabine in breast cancer patients with HER2-negative pathologic residual invasive disease after neoadjuvant chemotherapy (CREATE-X, JBCRG-04), San Antonio Breast Cancer Symposium. 2015. Abstract S1-07.

Submit your next manuscript to BioMed Central and we will help you at every step:

- We accept pre-submission inquiries

- Our selector tool helps you to find the most relevant journal

- We provide round the clock customer support

- Convenient online submission

- Thorough peer review

- Inclusion in PubMed and all major indexing services

- Maximum visibility for your research

Submit your manuscript at www.biomedcentral.com/submit
Biomed Central 